healthy donors: $0,0015(0,001-0,003)$ vs $0,003(0,001-0,007)$ and $0,01(0,005-$ $0,02)$ vs $0,02(0,01-0,04)$, respectively, $p<0,01$ for both cases. At baseline, a significant correlation was found in RA pts between absolute counts of memory $B$ cells $(C D 19+C D 27+)$ and $\operatorname{CRP}(r=0,50, p<0,05)$; the percentage and absolute counts of plasmablasts (CD19+CD38+++CD27+lgD-CD20-) and RF ( $r=0,41$ and $r=0,52, p<0,05)$. After 12 mo of TCZ therapy, $54 \%$ of pts were categorized as good responders, $46 \%$ of pts - as moderate responders according to the EULAR response criteria. Reductions in the percentages and absolute counts of plasmablasts (CD19+CD38+++CD27+lgD-CD20-) were documented after $12 \mathrm{mo}$ of TCZ therapy: $0,15 \%(0,1-0,3)$ vs $0,1 \%(0,01-0,1)$ and $0,0003(0,00007-0,004)$ vs $0,0001(0-0,0003)$, respectively, $p<0,05$. The median percentages/absolute counts of switched memory B cells (CD19+CD27+lgD-) were 6,8\%(3,6-11,6)/ $0,01(0,005-0,02)$ at baseline; and 3,1\%(1,1-4,2)/0,003(0,002-0,006) after 12 mo of TCZ therapy, $p>0,05$. After 12 mo of TCZ therapy, the median percentages and absolute counts of memory B cells (CD19+CD27+) and switched memory B cells (CD19+CD27+lgD-) became lower in RA pts than in the controls: $1,0 \%(0,7-1,2)$ vs $2,2 \%(1,1-3,0) ; 0,001(0,006-0,003)$ vs $0,003(0,001-0,007) ; 3,1 \%(1,1-4,2)$ vs $12,8 \%(9,3-17,0) ; 0,003(0,002-0,006)$ vs $0,02(0,01-0,04)$, respectively, $p<0,05$, respectively, for all cases. Other B-cell subpopulations did not changed after 12 mo of TCZ therapy as compared to baseline values.

Conclusions: Immunophenotyping in pts with active RA showed the decrease in the absolute counts of memory B cells (CD19+CD27+), switched memory B cells (CD19+ CD27+lgD-) as compared to healthy subjects. Positive correlation between the counts of memory $B$ cells and plasmablasts and values of laboratory indicators of RA (CRP, RF) suggests that B-lymphocytes may be involved in RA pathogenesis. The reduction in the levels of plasmonoblasts after $12 \mathrm{mo}$ of TCZ therapy was observed.

Disclosure of Interest: None declared

DOI: 10.1136/annrheumdis-2018-eular.4566

\section{SAT0180 1 TWO-YEAR CONSOLIDATED SAFETY DATA FOR ABP 501 IN PATIENTS WITH MODERATE TO SEVERE RHEUMATOID ARTHRITIS}

E. Krishnan ${ }^{1}$, N. Zhang ${ }^{1}$, H. Wang ${ }^{1}{ }^{1}$ Biosimilars Development, Amgen Inc., Thousand Oaks, United States

Background: Biosimilars are expected to have similar long-term safety profiles as originator products.

Objectives: To describe the consolidated, 2-year safety data on ABP 501, an approved biosimilar to adalimumab.

Methods: We combined individual patient data from a 26 -week randomized controlled head-to-head study (parent study) comparing ABP 501 with adalimumab (NCT01970475) and its 72-week open-label extension (OLE) study (NCT02114931) in which all patients received only ABP 501. Safety data were reported by exposure-adjusted incidence rate as the number of subjects with the specified adverse events *(AEs) per 100 person-years. AEs from the parent and OLE studies were summarized; for each category, patients were included only once based on the 1st event in that AE category. All comparisons were performed descriptively.

Results: In the parent study, 264 patients received ABP 501 and 262 patients received adalimumab reference product (RP). Of these, 229 in the ABP $501 \mathrm{arm}$ and 237 in the RP arm entered and were treated in the open-label extension study. The exposure-adjusted incidence rate for treatment-emergent AEs by treatment group are shown in the Table.

\begin{tabular}{|c|c|c|}
\hline & $\begin{array}{l}\text { ABP 501/ABP } 501 \\
(N=264)\end{array}$ & $\begin{array}{l}\text { Adalimumab RP/ABP } 501 \\
(\mathrm{~N}=262)\end{array}$ \\
\hline Adverse Event Category & \multicolumn{2}{|c|}{ Exposure-adjusted incidence rate $n / E(r)$} \\
\hline Any AE & $187 / 187.6(99.7)$ & 197/192.2 (102.5) \\
\hline Any grade $\geq 3 \mathrm{AE}$ & $32 / 405.2(7.9)$ & $30 / 410.6(7.3)$ \\
\hline Any treatment-related $\mathrm{AE}$ & $72 / 345.3(20.9)$ & $79 / 344.3(22.9)$ \\
\hline Any grade $\geq 3$ treatment-related $\mathrm{AE}$ & 6/427.2 (1.4) & $5 / 433.6(1.2)$ \\
\hline Any serious $\mathrm{AE}$ & $34 / 407.6(8.3)$ & $32 / 410.1(7.8)$ \\
\hline Any treatment-related serious $\mathrm{AE}$ & $6 / 427.9(1.4)$ & $2 / 434.3(0.5)$ \\
\hline Any events of interest & $141 / 261.5(53.9)$ & $154 / 254.7(60.5)$ \\
\hline Infections and infestations & $125 / 289.9(43.1)$ & 130/287.4 (45.2) \\
\hline Liver enzyme elevations & $25 / 401.4(6.2)$ & $20 / 415.6(4.8)$ \\
\hline Hypersensitivity & $19 / 407.7(4.7)$ & $22 / 412.2(5.3)$ \\
\hline Injection site reactions & $6 / 417.9(1.4)$ & $13 / 418.3(3.1)$ \\
\hline Hematological reactions & $6 / 421.8(1.4)$ & $7 / 426.1(1.6)$ \\
\hline Malignancies & $6 / 423.9(1.4)$ & $3 / 433.6(0.7)$ \\
\hline Heart failure & $1 / 428.4(0.2)$ & $2 / 435.6(0.5)$ \\
\hline
\end{tabular}

Conclusions: Over the 2-year observation period, there were no meaningful differences in AEs between adalimumab reference product and ABP 501.

Disclosure of Interest: E. Krishnan Shareholder of: Amgen Inc., Employee of: Amgen Inc., N. Zhang Shareholder of: Amgen Inc., Employee of: Amgen Inc., H. Wang Shareholder of: Amgen Inc., Employee of: Amgen Inc.

DOI: 10.1136/annrheumdis-2018-eular.6746

\section{SAT0181 EFFECT OF ANTI-IL-6 THERAPY ON SERUM LEVELS OF METABOLIC SYNDROME-RELATED BIOMARKERS IN RHEUMATOID ARTHRITIS PATIENTS}

F. Genre ${ }^{1}$, S. Remuzgo-Martínez ${ }^{1}$, R. López-Mejías ${ }^{1}$, V. Mijares ${ }^{1}$, J. Calvo-Alen ${ }^{2}$, R. Blanco ${ }^{1,3}$, J. Llorca ${ }^{4}$, S. Castañeda ${ }^{5}$, M. Á. González-Gay ${ }^{1,3,6,7}$. 'Epidemiology, Genetics and Atherosclerosis Research Group on Systemic Inflammatory Diseases, IDIVAL, Santander, ${ }^{2}$ Rheumatology Department, Hospital Universitario Araba, Vitoria, ${ }^{3}$ Rheumatology Department, Hospital Universitario Marqués de Valdecilla, ${ }^{4}$ Epidemiology and Computational Biology Department, School of Medicine, University of Cantabria, and CIBER Epidemiología y Salud Pública (CIBERESP), IDIVAL, Santander, ${ }^{5}$ Rheumatology Department, Hospital Universitario La Princesa, IIS-IPrincesa, Madrid, ${ }^{6}$ School of Medicine, University of Cantabria, Santander, Spain, ${ }^{7}$ Cardiovascular Pathophysiology and Genomics Research Unit, School of Physiology, Faculty of Health Sciences, University of the Witwatersrand, Johannesburg, South Africa

Background: Metabolic syndrome (MeS) is a pathologic state that encompasses metabolic anomalies such as hyperglycemia, dyslipidemia, obesity and hypertension and that, apart from being a cardiovascular risk factor, it has been associated with chronic inflammatory diseases such as rheumatoid arthritis $(R A)^{1,2}$. Ghrelin and retinol binding protein-4 (RBP-4) are two biomarkers associated with MeS, and are also linked to different cardiometabolic risk factors. In this regard, it is known that ghrelin exerts an anti-inflammatory role, while RBP-4 has a pro-inflammatory role ${ }^{2}$.

Objectives: Since a beneficial effect on endothelial function has been reported for anti-IL-6 therapy ${ }^{3}$, we aimed to evaluate the effect of a single infusion of antiIL- 6 on the serum levels of ghrelin and RBP-4 in patients with RA.

Methods: Ghrelin and RBP-4 levels were measured in serum samples from 50 Spanish individuals with RA that fulfilled the 2010 classification criteria ${ }^{4}$, and that were under treatment with the anti-IL-6 monoclonal antibody Tocilizumab. Patients with diabetes mellitus or plasma glucose levels $>110 \mathrm{mg} / \mathrm{dL}$ were excluded. Blood samples were taken in the fasting state, immediately before (time 0 ) and after (time 60 minutes) Tocilizumab infusion.

Results: A significant increase in serum levels of ghrelin was observed after a single infusion of Tocilizumab (mean \pm standard deviation: $72.99 \pm 58.43 \mathrm{pg} / \mathrm{mL}$ versus $134.02 \pm 225.93 \mathrm{pg} / \mathrm{mL}$, before and after Tocilizumab infusion, $p=0.04$ ). Serum levels of RBP-4 were not affected by the administration of Tocilizumab (mean 土standard deviation: $23.48 \pm 13.99 \mu \mathrm{g} / \mathrm{mL}$ versus $20.90 \pm 15.54 \mu \mathrm{g} / \mathrm{mL}$, before and after Tocilizumab infusion, $\mathrm{p}=0.42$ ).

Conclusions: Our results show that ghrelin levels increase after a single infusion of Tocilizumab, supporting the hypothesis that IL-6 blockade has a rapid beneficia effect on factors associated with MeS and cardiovascular risk in RA patients. Hence, long-term treatment with anti-IL-6 may reduce the risk of developing cardiovascular disease in RA.

\section{REFERENCES}

[1] Best Pract Res Clin Rheumatol 2016;30(5):851-869.

[2] Biomed Res Int 2014;2014:860651

[3] Atherosclerosis 2011;219:734-36.

[4] Arthritis Rheum 2010;62:2569-81.

Acknowledgements: FG is a recipient of a Sara Borrell post-doctoral fellowship from the Instituto de Salud Carlos III (ISCIII)(Spain), co-funded by European Social Fund (ESF,"Investing in your future") (CD15/00095). SR-M is supported by funds of the RETICS Program (RIER) RD16/0012/0009 (ISCIII, co-funded by the European Regional Development Fund, ERDF). RL-M is a recipient of a Miguel Servet type I programme fellowship from the ISCIII, co-funded by ESF (CP16/ 00033). VM is supported by funds of a Miguel Servet type I programme (CP16/ 00033)(ISCIII, co-funded by ERDF).

Disclosure of Interest: None declared

DOI: 10.1136/annrheumdis-2018-eular.6829 